\title{
A Mild Synthesis of New Aryl Vinyl Ethers and Diethyl 1-[(Alkyl)(cyano)methyl] vinylphosphonates via the Substitution of a 2,3-Difunctional Allyl Bromide
}

\author{
Asma Fray, ${ }^{1}$ Jihène Ben Kraïem, ${ }^{2}$ Aïcha Arfaoui, ${ }^{1}$ and Hassen Amri ${ }^{1}$ \\ ${ }^{1}$ Selective Organic Synthesis \& Biological Activity, Faculty of Science, El Manar University, 2092 Tunis, Tunisia \\ ${ }^{2}$ Laboratoire Méthodes et Techniques d'Analyse (LMTA), Institut National de Recherche et d'Analyse Physico-Chimique \\ (INRAP) Biotechnopôle Sidi-Thabet, 2020 Tunis, Tunisia
}

Correspondence should be addressed to Hassen Amri; hassen.amri@fst.rnu.tn

Received 13 December 2013; Accepted 2 January 2014; Published 12 February 2014

Academic Editors: T. Mino and H. Miyabe

Copyright (C) 2014 Asma Fray et al. This is an open access article distributed under the Creative Commons Attribution License, which permits unrestricted use, distribution, and reproduction in any medium, provided the original work is properly cited.

A novel class of aryl vinyl ethers 3 and diethyl 3-cyano-3-alkylprop-1-en-2-ylphosphonates $\mathbf{4}$ has been prepared, respectively, from coupling reaction of diethyl 1-(bromomethyl)-2-cyanovinylphosphonate 2 with phenols and Gilman reagents.

\section{Introduction}

The development of new strategies for the synthesis of organic compounds containing an $\alpha$-bromomethyl group as in product 1 continues to pose a challenge to organic chemists. It has resulted in a wide variety of applications in the preparation of several natural [1-4] as well as biologically active compounds [5-8]. Moreover, the utility of these versatile allyl brominated intermediates 1 [9-18] comes from their reaction with different nucleophilic species and additionally their ability to act as excellent Michael acceptors. Because of its importance, it is still of interest to develop novel approaches for the efficient generation of functionalized allyl bromide $\mathbf{1}$. In this regard, we have previously described a simple and stereoselective synthesis of diethyl (E)-1-(bromomethyl)-2cyanovinylphosphonate 2 [19], an activated alkene bearing three functional groups. We have also demonstrated that the latter can be used as an efficient electrophilic synthon for the synthesis of a new family of allylamines [19], enamines [20], and vinyl ether [20]. Prompted by the versatility of allyl bromide 2 , we suggested that its reaction with a variety of phenols and Gilman reagents would be a very convenient way to prepare new substituted aryl vinyl ethers 3 and diethyl 1[(alkyl)(cyano)methyl] vinylphosphonates 4 (Scheme 1).

\section{Results and Discussion}

Functional aryl vinyl ethers have been shown to be promising building blocks in organic synthesis [21-23] as well as important intermediates for the synthesis of a range of products [24-33], new polymeric materials [34-36], and biologically active molecules [37]. Thus, the preparation of these kinds of molecules has always attracted the attention of organic chemists and a growing effort has been directed toward the development of new vinyl ether-based structures and new methods for their construction [38-49]. Examination of the synthetic approaches to a wide variety of vinyl ethers has shown serious limitations, such as the use of strong acids or bases, toxic metals, and high temperatures. Therefore, the need to implement new inexpensive and easy methodologies to produce functional vinyl ethers remains attractive. In this context, we expected that the substitution of the bromine atom in molecule $\mathbf{2}$ by aromatic alcohols as nucleophilic reagents might lead to the desired aryl vinyl ethers 3 . Hence, condensation of allyl bromide 2 with phenol (1.0 equiv.) in the presence of potassium carbonate (1.2 equiv.) and in refluxing acetonitrile afforded the corresponding aryl vinyl ether $\mathbf{3 a}$ in $92 \%$ yield after $15 \mathrm{~min}$. In order to generalize the reaction of allyl bromide 2 with various aromatic alcohols, we examined 

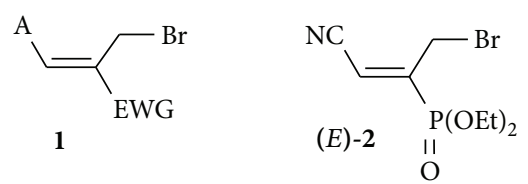
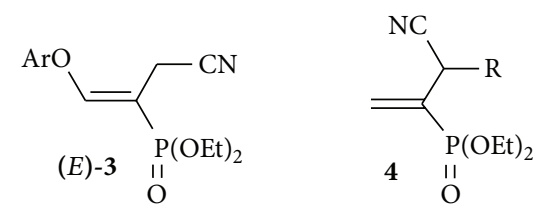

$\mathrm{A}=\mathrm{H}, \mathrm{R}, \mathrm{CO}_{2} \mathrm{R}, \quad \mathrm{EWG}=\mathrm{CO}_{2} \mathrm{R}, \mathrm{CN}, \mathrm{CO}_{2} \mathrm{H}, \mathrm{COR}, \mathrm{P}(\mathrm{O})(\mathrm{OEt})_{2}$

Scheme 1: Target functional vinylphosphonates $\mathbf{3}$ and $\mathbf{4}$ obtained from allyl bromide 2 .

TABLE 1: Synthesis of functional aryl vinyl ethers $\mathbf{3 a - f .}$

\begin{tabular}{lccc}
\hline Product & Ar & Time $(\min )$ & ${\text { Yield }(\%)^{\mathrm{a}}}^{\mathrm{a}}$ \\
\hline 3a & Phenyl & 15 & 92 \\
3b & 4-Methylphenyl & 15 & 68 \\
3c & 3-Hydroxyphenyl & 30 & 84 \\
3d & 4-Nitrophenyl & 15 & 88 \\
3e & 3-Methoxyphenyl & 45 & 78 \\
3f & 2,4,6-Tribromophenyl & 15 & 57 \\
\hline
\end{tabular}

${ }^{\text {a }}$ Yields refer to isolated products characterized by ${ }^{1} \mathrm{H},{ }^{13} \mathrm{C}$ NMR spectroscopy.

a variety of electronically and sterically substituted phenols. As shown in Table 1, the reaction proceeded smoothly regardless of the steric and electronic properties of these different phenols. With few exceptions, the reaction was generally carried out in 15 minutes in good to excellent yields (Scheme 2, Table 1).

The formation of vinyl ethers $\mathbf{3 a}-\mathbf{f}$ is the result of the $\mathrm{S}_{\mathrm{N}} 2$ substitution of allyl bromide $\mathbf{2}$ by different aryloxy groups followed by isomerization. A plausible reaction pathway for the synthesis of these new trisubstituted alkenes $\mathbf{3 a - f}$ is shown in Scheme 3.

The structures of $\mathbf{3} \mathbf{a}-\mathbf{f}$ were established on the basis of their ${ }^{1} \mathrm{H}$ and ${ }^{13} \mathrm{C}$ NMR spectra and by heteronuclear multiple bond correlation (HMBC). Their stereochemistry has been assigned on the basis of NOESY experiment. A sample of $\mathbf{3 a}$ shows no correlation between the vinylic proton $(\delta: 7.53 \mathrm{ppm})$ and those of $\mathrm{CH}_{2} \mathrm{CN}(\delta: 3.34 \mathrm{ppm})$. This result indicates that the vinylic proton and the $\mathrm{CH}_{2}$ group are on opposite sides of the double bond and therefore the alkene in $\mathbf{3 a}$ is $E$ configuration.

This result allowed the consideration of other experimental goals to exploit the electrophilicity of allyl bromide 2 by examining its reactivity with other nucleophilic reagents such as organocuprates. Gilman reagents have been widely used for the construction of organic molecules [50-53], since their discovery in 1900. They display an excellent reactivity towards a wide range of electrophiles and readily undergo transmetallation to provide a variety of organometallic species as organocopper derivatives which react well with soft electrophiles and display excellent chemoselectivity [54]. As a follow-up of our research in the direct substitution of functionalized allyl bromide by organocuprates reagents [55], diethyl (E)-1-(bromomethyl)-2-cyanovinylphosphonate 2 constituted the ideal intermediate for the synthesis of new 2cyanoethylphosphonates 4. As shown in Scheme 4, conjugate addition of dialkyl organocuprates, generated in situ at low temperature from Grignard reagents in the presence of
$\mathrm{LiCuBr}_{2}$, leads to the corresponding Michael acceptors 4 in an $\mathrm{S}_{\mathrm{N}} 2^{\prime}$ substitution process. The new vinylphosphonates 4 obtained are summarized in Table 2.

\section{Conclusion}

In summary, a practical and efficient synthesis of new substituted aryl vinyl ethers 3 and diethyl 1-[(alkyl)(cyano)methyl] vinylphosphonates 4 has been developed. The application of diethyl (E)-1-(bromomethyl)-2-cyanovinylphosphonate $\mathbf{2}$ in other processes will be communicated in due course.

\section{Experimental Section}

4.1. Materials and Instrumentation. Starting materials and solvents were purchased and used without further purification. ${ }^{1} \mathrm{H}-\mathrm{NMR}$ and ${ }^{13} \mathrm{C}-\mathrm{NMR}$ spectra were recorded on a Bruker AMX 300 spectrometer working at $300 \mathrm{MHz}$, $121 \mathrm{MHz}$, and $75 \mathrm{MHz}$, respectively, for ${ }^{1} \mathrm{H},{ }^{31} \mathrm{P}$, and ${ }^{13} \mathrm{C}$ with $\mathrm{CDCl}_{3}$ as the solvent and TMS as the internal standard. The chemical shifts $(\delta)$ and coupling constants $(J)$ are, respectively, expressed in parts per million (ppm) and hertz $(\mathrm{Hz})$. All NMR spectra were acquired at $25^{\circ} \mathrm{C}$. Assignments of proton $\left({ }^{1} \mathrm{H}-\mathrm{NMR}\right)$ and carbon $\left({ }^{13} \mathrm{C}-\mathrm{NMR}\right)$ signals were secured by DEPT 135 and HMBC experiments. Multiplicity of peaks is indicated by the following: s, singlet; d, doublet; dd, doublet of doublets; $\mathrm{t}$, triplet; dt, doublet of triplets; q, quartet; dq, doublet of quartets; hept, heptuplet; m, multiplet. All reactions were monitored by TLC on silica gel plates (Fluka Kieselgel 60 F254, Merck) eluting with the solvents indicated and visualized by a $254 \mathrm{~nm}$ UV lamp and aqueous potassium permanganate solution. For column chromatography, Fluka Kieselgel 70-230 mesh was used. The compounds were examined by gas chromatography-mass spectrometry (GC/MS) and spectra were recorded on an Agilent Technologies $6890 \mathrm{~N}$ instrument with an Agilent $5973 \mathrm{~N}$ mass detector (EI) and an HP5-MS $30 \mathrm{~m} \times 0.25 \mathrm{~mm}$ capillary apolar column (stationary 


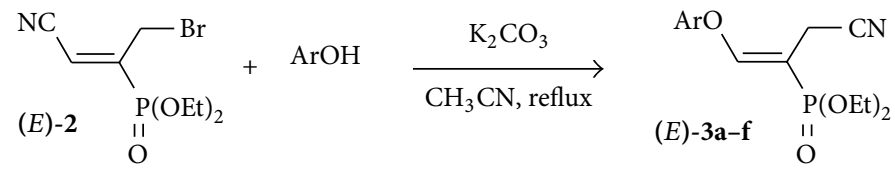

Scheme 2: Basic $O$-vinylation of phenols with allyl bromide 2.

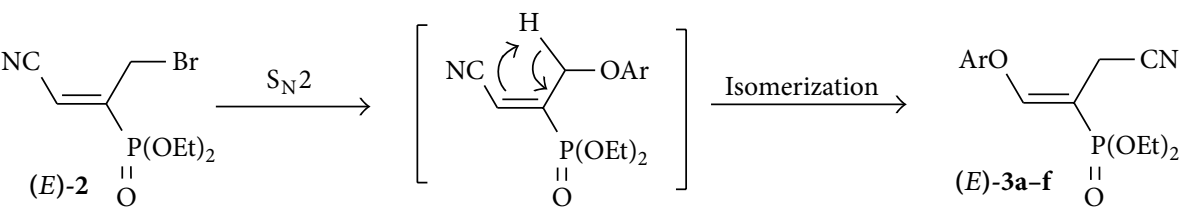

Scheme 3: Isomerization of allyl ethers to the corresponding vinyl ones 3.

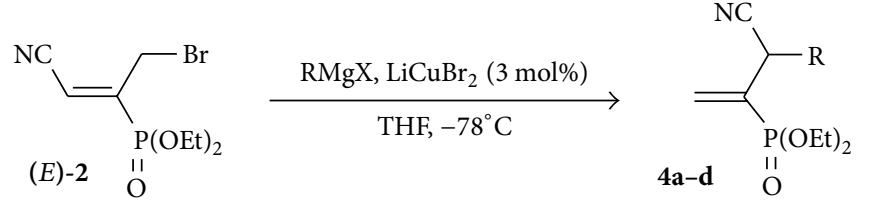

SCHEME 4: Conjugate addition of Gilman regents on allyl bromide 2.

phase: $5 \%$ diphenyldimethylpolysiloxane film, $0.25 \mu \mathrm{m})$. rt indicates the retention time. IR spectra were recorded on a Bruker Vertex 70 FT-IR spectrophotometer. The elementary analyses $(\mathrm{C}, \mathrm{H}$, and $\mathrm{N})$ were performed on a Perkin-Elmer Series II CHNS/O Analyzer 2400.

4.2. General Procedure for the Synthesis of Aryl Vinyl Ethers $(\mathbf{3} \boldsymbol{a}-\boldsymbol{f})$. A mixture of allyl bromide $(\boldsymbol{E})-\mathbf{1}(282 \mathrm{mg}$, $1.0 \mathrm{mmol})$, phenol $(1.0 \mathrm{mmol})$, and powdered anhydrous $\mathrm{K}_{2} \mathrm{CO}_{3}(165 \mathrm{mg}, 1.2 \mathrm{mmol})$ in acetonitrile $(3 \mathrm{~mL})$ was refluxed for 15 to 45 minutes under a nitrogen atmosphere with stirring. The reaction mixture was allowed to cool and evaporated to dryness, and the residue was extracted with ethyl acetate $(3 \times 20 \mathrm{~mL})$ and then the organic layer was washed with brine $(20 \mathrm{~mL})$, dried over $\mathrm{MgSO}_{4}$, and concentrated under reduced pressure. The obtained liquid was purified by column chromatography (Hexane-AcOEt, 1:1).

4.2.1. Diethyl (E)-[1-Cyanomethyl-2-(phenoxy)]vinylphosphonate (3a). Yellow liquid. Yield: 92\%, IR (neat): 3011, 2225, 1713, 1455, $1242 \mathrm{~cm}^{-1}$. ${ }^{1} \mathrm{H}-\mathrm{NMR}\left(300 \mathrm{MHz}, \mathrm{CDCl}_{3}\right): 7.53$ (d, $\left.1 \mathrm{H},{ }^{3} J_{H P}=12 \mathrm{~Hz},=\mathrm{CH}\right) ; 7.38(\mathrm{t}, 2 \mathrm{H}, J=9 \mathrm{~Hz}$, aromatic $\mathrm{H}) ; 7.20$ $(\mathrm{t}, 1 \mathrm{H}, J=9 \mathrm{~Hz}$, aromatic $\mathrm{H}) ; 7.09(\mathrm{~d}, 2 \mathrm{H}, J=9 \mathrm{~Hz}$, aromatic $\mathrm{H}) ; 4.15\left(\mathrm{dq}, J=7.5 \mathrm{~Hz}, J=7.5 \mathrm{~Hz}, 4 \mathrm{H}, 2 \mathrm{OCH}_{2}\right) ; 3.34(\mathrm{~d}$, $\left.2 \mathrm{H},{ }^{3} \mathrm{~J}_{\mathrm{HP}}=15 \mathrm{~Hz}, \mathrm{CH}_{2}\right) ; 1.38\left(\mathrm{t}, 6 \mathrm{H}, \mathrm{J}=7.5 \mathrm{~Hz}, 2 \mathrm{CH}_{3}\right) ;{ }^{13} \mathrm{C}-$ $\operatorname{NMR}\left(75 \mathrm{MHz}, \mathrm{CDCl}_{3}\right): 157.0\left(\mathrm{~d},=\mathrm{CH},{ }^{2} J_{\mathrm{CP}}=27.75 \mathrm{~Hz}\right) ; 156.1$ (aromatic C); 130.0 (aromatic $\mathrm{CH}) ; 125.2$ (aromatic $\mathrm{CH}) ; 117.6$ (aromatic CH); $116.6\left(\mathrm{~d}, \mathrm{CN},{ }^{3} J_{\mathrm{CP}}=3.75 \mathrm{~Hz}\right) ; 98.4(\mathrm{~d},=\mathrm{C}$, $\left.{ }^{1} J_{C P}=201 \mathrm{~Hz}\right) ; 62.3\left(\mathrm{~d}, 2 \mathrm{OCH}_{2},{ }^{2} J_{C P}=5.25 \mathrm{~Hz}\right) ; 16.2(\mathrm{~d}$, $\left.2 \mathrm{CH}_{3},{ }^{3} J_{\mathrm{CP}}=6.75 \mathrm{~Hz}\right) ; 12.9\left(\mathrm{~d}, \mathrm{CH}_{2},{ }^{2} J_{\mathrm{CP}}=6 \mathrm{~Hz}\right) ;{ }^{31} \mathrm{P}-$ NMR (121 MHz, $\mathrm{CDCl}_{3}$ ): 17.70; GC/MS (EI): rt = $32.21 \mathrm{~min}$, $m / z=295$ (M+, 54), 239 (58), 94 (100), 77 (78), 65 (55) 51
(36). Anal. calcd for $\mathrm{C}_{14} \mathrm{H}_{18} \mathrm{NO}_{4} \mathrm{P}(295,10)$ : C, 56.95; $\mathrm{H}, 6.14$; $\mathrm{N}, 4.74$. Found: C, 56.67; H, 5.04; N, 4.03.

4.2.2. Diethyl (E)-[1-Cyanomethyl-2-(4-methylphenoxy)]vinylphosphonate (3b). Yellow liquid. Yield: $68 \%$, IR (neat): 3015, 2222, 1715, 1456, $1245 \mathrm{~cm}^{-1}$. ${ }^{1} \mathrm{H}-\mathrm{NMR}\left(300 \mathrm{MHz} \mathrm{CDCl}_{3}\right): 7.49$ $\left(\mathrm{d}, 1 \mathrm{H},{ }^{3} J_{H P}=12 \mathrm{~Hz},=\mathrm{CH}\right) ; 7.15(\mathrm{~d}, 2 \mathrm{H}, J=9 \mathrm{~Hz}$, aromatic $\mathrm{H})$; $6.98(\mathrm{~d}, 2 \mathrm{H}, J=9 \mathrm{~Hz}$, aromatic $\mathrm{H}) ; 4.15(\mathrm{dq}, 4 \mathrm{H}, J=7.5 \mathrm{~Hz}, 4 \mathrm{H}$, $\left.J=7.5 \mathrm{~Hz}, 2 \mathrm{OCH}_{2}\right) ; 3.33\left(\mathrm{~d}, 2 \mathrm{H},{ }^{3} J_{\mathrm{HP}}=15 \mathrm{~Hz}, \mathrm{CH}_{2}\right) ; 2.33(\mathrm{~s}$, $\left.3 \mathrm{H}, \mathrm{CH}_{3}\right) ; 1.38\left(\mathrm{t}, 6 \mathrm{H}, \mathrm{J}=7.5 \mathrm{~Hz}, 2 \mathrm{CH}_{3}\right) ;{ }^{13} \mathrm{C}-\mathrm{NMR}(75 \mathrm{MHz}$, $\mathrm{CDCl}_{3}$ ): $157.5\left(\mathrm{~d},=\mathrm{CH},{ }^{2} J_{\mathrm{CP}}=27.75 \mathrm{~Hz}\right) ; 154.1$ (aromatic C); 134.9 (aromatic C); 130.4 (aromatic $\mathrm{CH}) ; 117.2$ (aromatic $\mathrm{CH})$; $116.7\left(\mathrm{~d}, \mathrm{CN},{ }^{3} J_{\mathrm{CP}}=3.75 \mathrm{~Hz}\right) ; 97.8\left(\mathrm{~d},=\mathrm{C},{ }^{1} J_{\mathrm{CP}}=201 \mathrm{~Hz}\right)$; $62.8\left(\mathrm{~d}, 2 \mathrm{OCH}_{2},{ }^{2} J_{\mathrm{CP}}=6 \mathrm{~Hz}\right) ; 20.7\left(\mathrm{~s}, \mathrm{CH}_{3}\right) ; 16.3\left(\mathrm{~d}, 2 \mathrm{CH}_{3}\right.$, $\left.{ }^{3} J_{C P}=6 \mathrm{~Hz}\right) ; 12.9\left(\mathrm{~d}, \mathrm{CH}_{2},{ }^{2} J_{C P}=6 \mathrm{~Hz}\right) ;{ }^{31} \mathrm{P}-\mathrm{NMR}(121 \mathrm{MHz}$, $\mathrm{CDCl}_{3}$ ): 17.91; GC/MS (EI): $\mathrm{rt}=37.9 \mathrm{~min}, m / z=309(\mathrm{M}+$, 40), 202 (36), 146 (33), 108 (100), 91 (44), 77 (33), 65 (38). Anal. calcd for $\mathrm{C}_{15} \mathrm{H}_{20} \mathrm{NO}_{4} \mathrm{P}(309,11): \mathrm{C}, 58.25 ; \mathrm{H}, 6.52 ; \mathrm{N}$, 4.53. Found: C, 57.98; H, 5.62; N, 4.47.

4.2.3. Diethyl (E)-[1-Cyano-2-(3-hydroxyphenoxy)]vinylphosphonate (3c). Yellow liquid. Yield: 84\%, IR (neat): 3356, 3013, 2222, 1718, 1454, $1243 \mathrm{~cm}^{-1}$. ${ }^{1} \mathrm{H}-\mathrm{NMR}\left(300 \mathrm{MHz}, \mathrm{CDCl}_{3}\right)$ : 8.94 (br, s, $1 \mathrm{H}, \mathrm{OH}) ; 7.62\left(\mathrm{~d}, 1 \mathrm{H},{ }^{3} J_{H P}=12 \mathrm{~Hz},=\mathrm{CH}\right) ; 7.13(\mathrm{t}$, $1 \mathrm{H}, J=7.5 \mathrm{~Hz}$, aromatic $\mathrm{H}) ; 6.72(\mathrm{~s}, 1 \mathrm{H}$, aromatic $\mathrm{H}) ; 6.70(\mathrm{~d}$, $1 \mathrm{H}, J=9 \mathrm{~Hz}$, aromatic $\mathrm{H}) ; 6.56(\mathrm{~d}, 1 \mathrm{H}, J=9 \mathrm{~Hz}$, aromatic $\mathrm{H}) ; 4.16\left(\mathrm{dq}, 4 \mathrm{H}, J=7.5 \mathrm{~Hz}, J=7.5 \mathrm{~Hz}, 2 \mathrm{OCH}_{2}\right) ; 3.33(\mathrm{~d}, 2 \mathrm{H}$, $\left.{ }^{3} J_{H P}=15 \mathrm{~Hz}, \mathrm{CH}_{2}\right) ; 1.39\left(\mathrm{t}, 6 \mathrm{H}, J=7.5 \mathrm{~Hz}, 2 \mathrm{CH}_{3}\right) ;{ }^{13} \mathrm{C}-\mathrm{NMR}$ $\left(75 \mathrm{MHz} \mathrm{CDCl}_{3}\right): 158.5$ (aromatic C); $157.7\left(\mathrm{~d},=\mathrm{CH},{ }^{2} J_{\mathrm{CP}}=\right.$ $29.25 \mathrm{~Hz}$ ); 156.9 (aromatic C); 130.4 (aromatic $\mathrm{CH}) ; 116.6$ (d, $\mathrm{CN},{ }^{3} J_{C P}=3.75 \mathrm{~Hz}$ ); 112.3 (aromatic $\mathrm{CH}$ ); 108.6 (aromatic $\mathrm{CH}) ; 104.4$ (aromatic CH); $96.8\left(\mathrm{~d},=\mathrm{C},{ }^{1} J_{C P}=203.25 \mathrm{~Hz}\right) ; 62.8$ 
TABLE 2: Synthesis of diethyl 1-[(alkyl)(cyano)methyl]vinylphosphonates $\mathbf{4 a - d . ~}$

\begin{tabular}{lcc}
\hline Product & $\operatorname{RMgX~(equiv.)~}$ & Yield (\%) $^{\mathrm{a}}$ \\
\hline 4a & $\operatorname{EtMgBr}(1.5)$ & 74 \\
4b & ${ }^{i} \operatorname{PrMgBr}(1.5)$ & 92 \\
4c & ${ }^{i} \operatorname{BuMgBr}(2)$ & 88 \\
4d & $\mathrm{Me}_{3} \mathrm{SiCH}_{2} \mathrm{MgCl}(1.5)$ & 78 \\
\hline
\end{tabular}

${ }^{\mathrm{a}}$ Isolated yields obtained after purification by chromatography.

$\left(\mathrm{d}, 2 \mathrm{OCH}_{2},{ }^{2} J_{\mathrm{CP}}=5.25 \mathrm{~Hz}\right) ; 16.2\left(\mathrm{~d}, 2 \mathrm{CH}_{3},{ }^{3} J_{\mathrm{CP}}=6.75 \mathrm{~Hz}\right)$; $12.8\left(\mathrm{CH}_{2},{ }^{2} J_{\mathrm{CP}}=6 \mathrm{~Hz}\right) ;{ }^{31} \mathrm{P}-\mathrm{NMR}\left(121 \mathrm{MHz}, \mathrm{CDCl}_{3}\right): 18.83$; GC/MS (EI): $\mathrm{rt}=38.93 \mathrm{~min}, m / z=311(\mathrm{M}+, 52), 146(30), 110$ (100), 81 (35), 65 (36). Anal. calcd for $\mathrm{C}_{14} \mathrm{H}_{18} \mathrm{NO}_{5} \mathrm{P}(311,09)$ : C, 54.02; H, 5.83; N, 4.50. Found: C, 53.81; H, 5.75; N, 4.45.

4.2.4. Diethyl (E)-[1-Cyanomethyl-2-(4-nitrophenoxy)]vinylphosphonate (3d). Orange liquid. Yield: 88\%, IR (neat): 3012, 2220, 1721, 1538, 1456, $1235 \mathrm{~cm}^{-1}$. ${ }^{1} \mathrm{H}-\mathrm{NMR}(300 \mathrm{MHz}$, $\left.\mathrm{CDCl}_{3}\right): 8.29(\mathrm{~d}, 2 \mathrm{H}, J=9 \mathrm{~Hz}$, aromatic $\mathrm{H}) ; 7.61\left(\mathrm{~d}, 1 \mathrm{H},{ }^{3} J_{\mathrm{HP}}=\right.$ $12 \mathrm{~Hz},=\mathrm{CH}) ; 7.28(\mathrm{~d}, 2 \mathrm{H}, J=9 \mathrm{~Hz}$, aromatic $\mathrm{H}) ; 4.19(\mathrm{dq}, 4 \mathrm{H}$, $\left.J=7.5 \mathrm{~Hz}, 4 \mathrm{H}, J=7.5 \mathrm{~Hz}, 2 \mathrm{OCH}_{2}\right) ; 3.39\left(\mathrm{~d}, 2 \mathrm{H},{ }^{3} J_{H P}=15 \mathrm{~Hz}\right.$, $\left.\mathrm{CH}_{2}\right) ; 1.40\left(\mathrm{t}, 6 \mathrm{H}, J=7.5 \mathrm{~Hz}, 2 \mathrm{CH}_{3}\right) ;{ }^{13} \mathrm{C}-\mathrm{NMR}(75 \mathrm{MHz}$, $\mathrm{CDCl}_{3}$ ): 159.8 (aromatic C); $154.2\left(\mathrm{~d},=\mathrm{CH},{ }^{2} J_{\mathrm{CP}}=27.75 \mathrm{~Hz}\right)$; 144.7 (aromatic C); 126.1 (aromatic $\mathrm{CH}$ ); 117.5 (aromatic $\mathrm{CH}$ ); $116.1\left(\mathrm{~d}, \mathrm{CN},{ }^{3} J_{C P}=3.75 \mathrm{~Hz}\right) ; 102.2\left(\mathrm{~d},=\mathrm{C},{ }^{1} J_{C P}=198 \mathrm{~Hz}\right)$; $62.8\left(\mathrm{~d}, 2 \mathrm{OCH}_{2},{ }^{2} J_{\mathrm{CP}}=6 \mathrm{~Hz}\right) ; 16.3\left(\mathrm{~d}, 2 \mathrm{CH}_{3},{ }^{3} J_{\mathrm{CP}}=6.75 \mathrm{~Hz}\right)$; $13.1\left(\mathrm{~d}, \mathrm{CH}_{2},{ }^{2} J_{\mathrm{CP}}=6 \mathrm{~Hz}\right) ;{ }^{31} \mathrm{P}-\mathrm{NMR}\left(121 \mathrm{MHz}, \mathrm{CDCl}_{3}\right): 16.17$; GC/MS (EI): $\mathrm{rt}=44.43 \mathrm{~min}, m / z=340(\mathrm{M}+, 38), 295(50)$, 267 (100), 146 (29), 109 (27), 81 (44), 66 (34). Anal. calcd for $\mathrm{C}_{14} \mathrm{H}_{17} \mathrm{~N}_{2} \mathrm{O}_{6} \mathrm{P}(340,08): \mathrm{C}, 49.42 ; \mathrm{H}, 5.04 ; \mathrm{N}, 8.23$. Found: $\mathrm{C}$, 49.20; H, 4.97; N, 8.13.

4.2.5. Diethyl (E)-[1-Cyanomethyl-2-(3-methoxyphenoxy)] vinylphosphonate (3e). Yellow liquid. Yield: 78\%, IR (neat): 3012, 2220, 1720, 1452, 1248, $1037 \mathrm{~cm}^{-1}$. ${ }^{1} \mathrm{H}-\mathrm{NMR}(300 \mathrm{MHz}$, $\left.\mathrm{CDCl}_{3}\right): 7.52\left(\mathrm{~d}, 1 \mathrm{H},{ }^{3} J_{\mathrm{HP}}=12 \mathrm{~Hz},=\mathrm{CH}\right) ; 7.26(\mathrm{t}, 1 \mathrm{H}, J=7.5 \mathrm{~Hz}$, aromatic $\mathrm{H}) ; 6.74(\mathrm{~d}, 1 \mathrm{H}, J=9 \mathrm{~Hz}$, aromatic $\mathrm{H}) ; 6.68(\mathrm{~d}, 1 \mathrm{H}, J$ $=9 \mathrm{~Hz}$, aromatic $\mathrm{H}) ; 6.64(\mathrm{~s}, 1 \mathrm{H}$, aromatic $\mathrm{H}) ; 4.16(\mathrm{dq}, 4 \mathrm{H}, J$ $\left.=7.5 \mathrm{~Hz}, J=7.5 \mathrm{~Hz}, 2 \mathrm{OCH}_{2}\right) ; 3.81\left(\mathrm{~s}, 3 \mathrm{H}, \mathrm{OCH}_{3}\right) ; 3.33(\mathrm{~d}, 2 \mathrm{H}$, $\left.{ }^{3} J_{\mathrm{HP}}=15 \mathrm{~Hz}, \mathrm{CH}_{2}\right) ; 1.39\left(\mathrm{t}, 6 \mathrm{H}, J=7.5 \mathrm{~Hz}, 2 \mathrm{CH}_{3}\right) ;{ }^{13} \mathrm{C}-\mathrm{NMR}$ $\left(75 \mathrm{MHz}, \mathrm{CDCl}_{3}\right.$ ): 161.0 (aromatic C); 157.0 (aromatic C); $156.9\left(\mathrm{~d},=\mathrm{CH},{ }^{2} J_{\mathrm{CP}}=27.75 \mathrm{~Hz}\right) ; 130.5($ aromatic $\mathrm{CH}) ; 116.6$ $\left(\mathrm{d}, \mathrm{CN},{ }^{3} J_{\mathrm{CP}}=3.75 \mathrm{~Hz}\right.$ ); 111.0 (aromatic $\mathrm{CH}$ ); 109.3 (aromatic $\mathrm{CH}) ; 103.7$ (aromatic $\mathrm{CH}) ; 98.4\left(\mathrm{~d},=\mathrm{C},{ }^{1} J_{\mathrm{CP}}=200.25 \mathrm{~Hz}\right)$; $62.3\left(\mathrm{~d}, 2 \mathrm{OCH}_{2},{ }^{2} J_{\mathrm{CP}}=5.25 \mathrm{~Hz}\right) ; 55.5\left(\mathrm{OCH}_{3}\right) ; 16.2\left(\mathrm{~d}, 2 \mathrm{CH}_{3}\right.$, $\left.{ }^{3} J_{C P}=6 \mathrm{~Hz}\right) ; 12.9\left(\mathrm{~d}, \mathrm{CH}_{2},{ }^{2} J_{C P}=6 \mathrm{~Hz}\right) ;{ }^{31} \mathrm{P}-\mathrm{NMR}(121 \mathrm{MHz}$, $\mathrm{CDCl}_{3}$ ): 17.64. Anal. calcd for $\mathrm{C}_{15} \mathrm{H}_{20} \mathrm{NO}_{5} \mathrm{P}(325,11)$ : C, 55.38; H, 6.20; N, 4.31. Found: C, 55.14; H, 6.14; N, 4.35.

4.2.6. Diethyl (E)-[1-Cyanomethyl-2-(2,4,6-tribromophenoxy)]vinylphosphonate (3f). White solid. Yield: 57\%, IR (neat): 3010, 2220, 1712, 1455, $1241 \mathrm{~cm}^{-1}$. ${ }^{1} \mathrm{H}-\mathrm{NMR}(300 \mathrm{MHz}$, $\mathrm{CDCl}_{3}$ ): $7.73(\mathrm{~s}, 2 \mathrm{H}$, aromatic $\mathrm{H}) ; 7.01\left(\mathrm{~d}, 1 \mathrm{H},{ }^{3} J_{\mathrm{HP}}=12 \mathrm{~Hz}\right.$, $=\mathrm{CH}) ; 4.14\left(\mathrm{dq}, 4 \mathrm{H}, J=7.5 \mathrm{~Hz}, 4 \mathrm{H}, J=7.5 \mathrm{~Hz}, 2 \mathrm{OCH}_{2}\right) ; 3.40$ $\left(\mathrm{d}, 2 \mathrm{H},{ }^{3} J_{\mathrm{HP}}=15 \mathrm{~Hz}, \mathrm{CH}_{2}\right) ; 1.39\left(\mathrm{t}, 6 \mathrm{H}, J=7.5 \mathrm{~Hz}, 2 \mathrm{CH}_{3}\right)$; ${ }^{13} \mathrm{C}-\mathrm{NMR}\left(75 \mathrm{MHz}, \mathrm{CDCl}_{3}\right): 157.5\left(\mathrm{~d},=\mathrm{CH},{ }^{2} \mathrm{~J}_{\mathrm{CP}}=28.5 \mathrm{~Hz}\right)$; 149.9 (aromatic C); 135.3 (aromatic $\mathrm{CH}$ ); 120.3 (aromatic C);
117.7 (aromatic C); $116.1\left(\mathrm{~d}, \mathrm{CN},{ }^{3} J_{\mathrm{CP}}=3.75 \mathrm{~Hz}\right) ; 100.0(\mathrm{~d}$, $\left.=\mathrm{C},{ }^{1} J_{C P}=198 \mathrm{~Hz}\right) ; 62.5\left(\mathrm{~d}, 2 \mathrm{OCH}_{2},{ }^{2} J_{\mathrm{CP}}=5.25 \mathrm{~Hz}\right) ; 16.2(\mathrm{~d}$, $\left.2 \mathrm{CH}_{3},{ }^{3} J_{\mathrm{CP}}=7.5 \mathrm{~Hz}\right) ; 12.9\left(\mathrm{~d}, \mathrm{CH}_{2},{ }^{2} J_{\mathrm{CP}}=5.25 \mathrm{~Hz}\right) ;{ }^{31} \mathrm{P}-\mathrm{NMR}$ $\left(121 \mathrm{MHz}, \mathrm{CDCl}_{3}\right): 16.35$; GC/MS (EI): $\mathrm{rt}=43.34 \mathrm{~min}, \mathrm{~m} / z=$ $531(\mathrm{M}+, 8), 452$ (44), 396 (100), 369 (38), 330 (22), 146 (52), 81 (72), 66 (42). Anal. calcd for $\mathrm{C}_{14} \mathrm{H}_{15} \mathrm{Br}_{3} \mathrm{NO}_{4} \mathrm{P}(528,83)$ : C, 31.61; H, 2.84; N, 2.63. Found: C, 31.57; H, 2.72; N, 2.59.

4.3. Representative Synthetic Procedure of Diethyl 1-[(Alkyl) (cyano)methyl]vinylphosphonates $(\mathbf{4 a}-\boldsymbol{d})$. To a mixture of diethyl (E)-1-bromomethyl-2-cyanovinylphsphonate $\mathbf{1}$ $(1.25 \mathrm{~g}, 5 \mathrm{mmol})$ and $1 \mathrm{M}$ solution of $\mathrm{LiCuBr}_{2}(0.15 \mathrm{~mL}, 3 \mathrm{~mol}$ $\%)$ in dry THF $(20 \mathrm{~mL})$ was added dropwise a solution of alkylmagnesium halide $(\mathrm{RMgX})$ at $\left(-78^{\circ} \mathrm{C}\right)$ under a nitrogen atmosphere. The resulting mixture was stirred for a few minutes and then quenched with saturated $\mathrm{NH}_{4} \mathrm{Cl}$ solution $(10 \mathrm{~mL})$ and extracted with ether $(3 \times 20 \mathrm{~mL})$. The combined organic layers were dried over $\mathrm{MgSO}_{4}$, filtered, and evaporated under reduced pressure. The crude product was purified by flash chromatography on silica gel $\left(\mathrm{Et}_{2} \mathrm{O} /\right.$ hexane, $9: 1)$ to afford diethyl 1-[(alkyl)(cyano) methyl]vinylphosphonates $4 \mathbf{a}-\mathbf{d}$.

4.3.1. Diethyl 3-Cyanopent-1-en-2-ylphosphonate (4a). Yellow Liquid. Yield: $74 \%$. IR (neat): 2226, $1689,1242 \mathrm{~cm}^{-1} \cdot{ }^{1} \mathrm{H}-\mathrm{NMR}$ $\left(300 \mathrm{MHz}_{\mathrm{CDCl}}\right): 6.30\left(\mathrm{~d}, 1 \mathrm{H},{ }^{3} J_{\mathrm{HP}}=21 \mathrm{~Hz},=\mathrm{CH}\right) ; 6.22(\mathrm{~d}$, $\left.1 \mathrm{H},{ }^{3} J_{\mathrm{HP}}=45 \mathrm{~Hz},=\mathrm{CH}\right) ; 4.12(\mathrm{dq}, 4 \mathrm{H}, J=7.5 \mathrm{~Hz}, J=7.5 \mathrm{~Hz}$, $\left.2 \mathrm{OCH}_{2}\right) ; 3.56\left(\mathrm{dt}, 1 \mathrm{H},{ }^{3} J_{\mathrm{HP}}=9 \mathrm{~Hz}, J=6 \mathrm{~Hz}, \mathrm{CH}\right) ; 2.00-1.77$ $\left(\mathrm{m}, 2 \mathrm{H}, \mathrm{CH}_{2}\right) ; 1.35\left(2 \mathrm{t}, 6 \mathrm{H}, J=7.5 \mathrm{~Hz}, J=7.5 \mathrm{~Hz}, 2 \mathrm{CH}_{3}\right) ; 1.09$ $\left(\mathrm{t}, 3 \mathrm{H}, J=7.5 \mathrm{~Hz}, \mathrm{CH}_{3}\right) ;{ }^{13} \mathrm{C}-\mathrm{NMR}\left(75 \mathrm{MHz}, \mathrm{CDCl}_{3}\right): 137.1$ (d, $\left.=\mathrm{C},{ }^{1} J_{\mathrm{CP}}=181.3 \mathrm{~Hz}\right) ; 132.1\left(\mathrm{~d},=\mathrm{CH}_{2},{ }^{2} J_{\mathrm{CP}}=8.25 \mathrm{~Hz}\right) ; 116.9(\mathrm{~d}$, $\left.\mathrm{CN},{ }^{3} J_{C P}=14.25 \mathrm{~Hz}\right) ; 62.4\left(\mathrm{~d}, 2 \mathrm{OCH}_{2},{ }^{2} J_{\mathrm{CP}}=6 \mathrm{~Hz}\right) ; 35.9(\mathrm{~d}$, $\left.\mathrm{CH},{ }^{2} J_{C P}=16.5 \mathrm{~Hz}\right) ; 25.9\left(\mathrm{~d}, \mathrm{CH}_{2},{ }^{3} J_{C P}=2.25 \mathrm{~Hz}\right) ; 16.1(\mathrm{~d}$, $\left.2 \mathrm{CH}_{3},{ }^{3} J_{\mathrm{CP}}=6 \mathrm{~Hz}\right) ; 10.9\left(\mathrm{CH}_{3}\right) ;{ }^{31} \mathrm{P}-\mathrm{NMR}\left(121 \mathrm{MHz}, \mathrm{CDCl}_{3}\right)$ : 15.54; GC/MS (EI): $\mathrm{rt}=42.81 \mathrm{~min}, m / z=231(\mathrm{M}+, 3), 167$ (29), 149 (100), 71 (19), 57 (34). Anal. calcd for $\mathrm{C}_{10} \mathrm{H}_{18} \mathrm{NO}_{3} \mathrm{P}$ (231,10): C, 51.94; H, 7.85; N, 6.06. Found: C, 51.69; H, 7.83; N, 6.03 .

4.3.2. Diethyl 3-Cyano-4-methylpent-1-en-2-ylphosphonate (4b). Yellow Liquid. Yield: 92\%. IR (neat): 2224, 1696, $1243 \mathrm{~cm}^{-1}$. ${ }^{1} \mathrm{H}-\mathrm{NMR}\left(300 \mathrm{MHz}, \mathrm{CDCl}_{3}\right): 6.31\left(\mathrm{~d}, 1 \mathrm{H},{ }^{3} J_{\mathrm{HP}}=\right.$ $21 \mathrm{~Hz},=\mathrm{CH}) ; 6.20\left(\mathrm{~d}, 1 \mathrm{H},{ }^{3} J_{H P}=45 \mathrm{~Hz},=\mathrm{CH}\right) ; 4.12(\mathrm{dq}, 4 \mathrm{H}, J$ $\left.=7.5 \mathrm{~Hz}, J=7.5 \mathrm{~Hz}, 2 \mathrm{OCH}_{2}\right) ; 3.57\left(\mathrm{dd}, 1 \mathrm{H},{ }^{3} J_{H P}=12 \mathrm{~Hz}, J=\right.$ $6 \mathrm{~Hz}, \mathrm{CH}) ; 2.28$ (hept, $1 \mathrm{H}, J=6 \mathrm{~Hz}, \mathrm{CH}) ; 1.34(\mathrm{t}, 6 \mathrm{H}, J=$ $\left.6 \mathrm{~Hz}, 2 \mathrm{CH}_{3}\right) ; 1.14\left(\mathrm{~d}, 3 \mathrm{H}, J=6 \mathrm{~Hz}, \mathrm{CH}_{3}\right) ; 0.98(\mathrm{~d}, 3 \mathrm{H}, J=$ $\left.6 \mathrm{~Hz}, \mathrm{CH}_{3}\right) ;{ }^{13} \mathrm{C}-\mathrm{NMR}\left(75 \mathrm{MHz}, \mathrm{CDCl}_{3}\right): 134.1\left(\mathrm{~d},=\mathrm{C},{ }^{1} J_{\mathrm{CP}}\right.$ $=176.25 \mathrm{~Hz}) ; 132.9\left(\mathrm{~d},=\mathrm{CH}_{2},{ }^{2} J_{\mathrm{CP}}=7.5 \mathrm{~Hz}\right) ; 117.7(\mathrm{~d}, \mathrm{CN}$, 
$\left.{ }^{3} J_{C P}=15.75 \mathrm{~Hz}\right) ; 62.5\left(\mathrm{~d}, 2 \mathrm{OCH}_{2},{ }^{2} J_{\mathrm{CP}}=12.75 \mathrm{~Hz}\right) ; 42.4(\mathrm{~d}$, $\left.\mathrm{CH},{ }^{2} J_{C P}=17.25 \mathrm{~Hz}\right) ; 29.4\left({ }^{3} J_{C P}=1.5 \mathrm{~Hz}, \mathrm{CH}\right) ; 21.5\left(\mathrm{CH}_{3}\right)$; $16.3\left(\mathrm{~d}, 2 \mathrm{CH}_{3},{ }^{3} J_{C P}=6 \mathrm{~Hz}\right) ;{ }^{31} \mathrm{P}-\mathrm{NMR}\left(121 \mathrm{MHz}, \mathrm{CDCl}_{3}\right)$ : 15.63; GC/MS (EI): $\mathrm{rt}=24.16 \mathrm{~min}, m / z=244(\mathrm{M}-1,3), 203$ (33), 175 (24), 147 (100), 81 (11), 66 (24). Anal. calcd for $\mathrm{C}_{11} \mathrm{H}_{20} \mathrm{NO}_{3} \mathrm{P}(245,12): \mathrm{C}, 53.87 ; \mathrm{H}, 8.22 ; \mathrm{N}, 5.71$. Found: $\mathrm{C}$, $53.54 ; \mathrm{H}, 7.48 ; \mathrm{N}, 5.79$.

4.3.3. Diethyl 3-Cyano-5-methylhex-1-en-2-ylphosphonate (4c). Colorless liquid. Yield: 88\%. IR (neat): 2226, 1693, $1244 \mathrm{~cm}^{-1}$. ${ }^{1} \mathrm{H}-\mathrm{NMR}\left(300 \mathrm{MHz}, \mathrm{CDCl}_{3}\right): 6.3\left(\mathrm{~d}, 1 \mathrm{H},{ }^{3} J_{\mathrm{HP}}=\right.$ $24 \mathrm{~Hz},=\mathrm{CH}) ; 6.23\left(\mathrm{dd}, 1 \mathrm{H},{ }^{3} J_{H P}=45 \mathrm{~Hz}, J=3 \mathrm{~Hz},=\mathrm{CH}\right)$; $4.12\left(\mathrm{dq}, 4 \mathrm{H}, J=7.5 \mathrm{~Hz}, J=7.5 \mathrm{~Hz}, 2 \mathrm{OCH}_{2}\right) ; 3.56(\mathrm{dt}, 1 \mathrm{H}$, ${ }^{3} J_{H P}=9 \mathrm{~Hz}, J=6 \mathrm{~Hz}, \mathrm{CH}$ ); 1.60 (hept, $1 \mathrm{H}, J=6 \mathrm{~Hz}, \mathrm{CH}$ ); 1.40-1.26 (m, 2H, CH $\left.)_{2}\right) ; .35(2 \mathrm{t}, 6 \mathrm{H}, J=7.5 \mathrm{~Hz}, J=7.5 \mathrm{~Hz}$, $\left.2 \mathrm{CH}_{3}\right) ; 0.92\left(2 \mathrm{~d}, 6 \mathrm{H}, J=6 \mathrm{~Hz}, 2 \mathrm{CH}_{3}\right) ;{ }^{13} \mathrm{C}-\mathrm{NMR}(75 \mathrm{MHz}$, $\left.\mathrm{CDCl}_{3}\right): 134.8\left(\mathrm{~d},=\mathrm{C},{ }^{1} J_{\mathrm{CP}}=181.5 \mathrm{~Hz}\right) ; 132.5\left(\mathrm{~d},=\mathrm{CH}_{2},{ }^{2} J_{\mathrm{CP}}=\right.$ $8.25 \mathrm{~Hz}) ; 119.3\left(\mathrm{~d}, \mathrm{CN},{ }^{3} J_{C P}=13.5 \mathrm{~Hz}\right) ; 62.5\left(\mathrm{~d}, 2 \mathrm{OCH}_{2},{ }^{2} J_{C P}=\right.$ $8.25 \mathrm{~Hz}) ; 35.7\left(\mathrm{~d}, \mathrm{CH}_{2},{ }^{3} J_{\mathrm{CP}}=1.5 \mathrm{~Hz}, \mathrm{CH}_{2}\right) ; 34.5\left(\mathrm{~d}, \mathrm{CH},{ }^{2} J_{C P}\right.$ $=16.5 \mathrm{~Hz}) ; 27.5(\mathrm{CH}) ; 22.5\left(\mathrm{CH}_{3}\right) ; 22.1\left(\mathrm{CH}_{3}\right) ; 16.2\left(\mathrm{~d}, 2 \mathrm{CH}_{3}\right.$, $\left.{ }^{3} J_{C P}=6.75 \mathrm{~Hz}\right) ;{ }^{31} \mathrm{P}-\mathrm{NMR}\left(121 \mathrm{MHz}, \mathrm{CDCl}_{3}\right): 15.65 ; \mathrm{GC} / \mathrm{MS}$ (EI): $\mathrm{rt}=28.04 \mathrm{~min}, m / z=272(\mathrm{M}-1,5), 233$ (43), 203 (32), 174 (30), 160 (19), 147 (100), 138 (27), 66 (36). Anal. calcd for $\mathrm{C}_{13} \mathrm{H}_{24} \mathrm{NO}_{3} \mathrm{P}(259,28): \mathrm{C}, 55.59 ; \mathrm{H}, 8.55 ; \mathrm{N}, 5.40$. Found: $\mathrm{C}$, 56.44; H, 8.43; N, 5.35 .

4.3.4. Diethyl 3-Cyano-4-(trimethylsilyl)but-1-en-2-ylphosphonate (4d). Yellow liquid. Yield: 78\%. IR (neat): 2223, 1711, $1242 \mathrm{~cm}^{-1}$. ${ }^{1} \mathrm{H}-\mathrm{NMR}\left(300 \mathrm{MHz}, \mathrm{CDCl}_{3}\right): 6.10\left(\mathrm{dd}, 1 \mathrm{H},{ }^{3} J_{\mathrm{HP}}=\right.$ $48 \mathrm{~Hz}, J=3 \mathrm{~Hz}=\mathrm{CH}) ; 6.08\left(\mathrm{~d}, 1 \mathrm{H},{ }^{3} J_{\mathrm{HP}}=21 \mathrm{~Hz},=\mathrm{CH}\right) ; 3.97$ $\left(\mathrm{dq}, 4 \mathrm{H}, J=7.5 \mathrm{~Hz}, J=7.5 \mathrm{~Hz}, 2 \mathrm{OCH}_{2}\right) ; 3.56(\mathrm{~m}, 1 \mathrm{H}, \mathrm{CH}) ; 1.96-$ $1.8\left(2 \mathrm{~m}, 2 \mathrm{H}, \mathrm{CH}_{2}\right) ; 1.20\left(2 \mathrm{t}, 6 \mathrm{H}, J=6 \mathrm{~Hz}, J=6 \mathrm{~Hz}, 2 \mathrm{CH}_{3}\right) ; 0.00$ $\left(\mathrm{s}, 9 \mathrm{H}, \mathrm{SiMe}_{3}\right) ;{ }^{13} \mathrm{C}-\mathrm{NMR}\left(75 \mathrm{MHz}, \mathrm{CDCl}_{3}\right): 139.2$ (d, =C, ${ }^{1} J_{\mathrm{CP}}$ $=180 \mathrm{~Hz}) ; 132.6\left(\mathrm{~d},=\mathrm{CH}_{2},{ }^{2} J_{\mathrm{CP}}=8.25 \mathrm{~Hz}\right) ; 121.8\left(\mathrm{~d}, \mathrm{CN},{ }^{3} J_{\mathrm{CP}}\right.$ $=12 \mathrm{~Hz}) ; 62.5\left(\mathrm{~d}, 2 \mathrm{OCH}_{2},{ }^{2} J_{\mathrm{CP}}=9 \mathrm{~Hz}\right) ; 29.4\left(\mathrm{~d}, \mathrm{CH},{ }^{2} J_{\mathrm{CP}}=\right.$ $15.75 \mathrm{~Hz}) ; 21.4\left(\mathrm{~d}, \mathrm{CH}_{2},{ }^{3} J_{\mathrm{CP}}=2.25 \mathrm{~Hz}\right) ; 16.2\left(\mathrm{~d}, 2 \mathrm{CH}_{3},{ }^{3} J_{C P}=\right.$ $6.75 \mathrm{~Hz}) ;-1.57\left(\mathrm{~s}, \mathrm{SiMe}_{3}\right) ;{ }^{31} \mathrm{P}-\mathrm{NMR}\left(121 \mathrm{MHz}, \mathrm{CDCl}_{3}\right): 15.69$. Anal. calcd for $\mathrm{C}_{12} \mathrm{H}_{24} \mathrm{NO}_{3}$ PSi $(289,13)$ : C, 49.81; H, 8.36; N, 4.84. Found: C, 49.60; H, 8.07; N, 4.76.

\section{Conflict of Interests}

The authors declare that there is no conflict of interests regarding the publication of this paper.

\section{Acknowledgments}

This work was supported by the Higher Education and Scientific Research of Tunisia. The authors wish to thank Professors Jacques Lebreton (University of Nantes, France) and Adel Nefzi (University of California, San Diego) for logistical help.

\section{References}

[1] F. Ameer, S. E. Drewes, N. D. Emslie, P. T. Kaye, and R. L. Mann, "Necic acid synthons. Part 2. Regioselectivity in the reactions of $(\mathrm{Z})$-2-bromomethyl-2-alkenoate esters with selected carbon nucleophiles," Journal of the Chemical Society, Perkin Transactions 1, vol. 28, pp. 2293-2295, 1983.

[2] H. M. R. Hoffmann and J. Rabe, "DABCO-catalyzed coupling of aldehydes with activated double bonds. 4. Stereoselective synthesis of trisubstituted olefins and terpenoid building blocks via 2-(hydroxyalkyl)-2-propenoic esters," Journal of Organic Chemistry, vol. 50, no. 20, pp. 3849-3859, 1985.

[3] H. M. R. Hoffmann and J. Rabe, "1, 4-Diazobicyclo[2. 2. 2] octane-catalyzed coupling of aldehydes and activated double bonds. Part 3. A short ans practical synthesis of mikanecic acid (4-vinyl-1-cyclohexene-1, 4-dicarboxylic acid)," Helvetica Chimica Acta, vol. 67, no. 2, pp. 413-415, 1984.

[4] H. M. R. Hoffmann and J. Rabe, "Preparation of 2-(1Hydroxyalkyl)acrylic esters, simple three-step synthesis of mikanecic acid," Angewandte Chemie International Edition, vol. 22, no. 10, pp. 795-796, 1983.

[5] T.-P. Loh and P.-L. Lye, "A concise synthesis of ( \pm )-methylenolactocin and the formal synthesis of $( \pm)$-phaseolinic acid," Tetrahedron Letters, vol. 42, no. 20, pp. 3511-3514, 2001.

[6] R. Buchholz and H. M. R. Hoffmann, " $\alpha$-methylidene- and $\alpha$-alkylidene- $\beta$-lactams from nonproteinogenic amino acids," Helvetica Chimica Acta, vol. 74, no. 6, pp. 1213-1220, 1991.

[7] M. Paira, B. Banerjee, S. Jana, S. K. Mandal, and S. C. Roy, "Titanocene(III) chloride mediated radical-induced one-pot synthesis of $\alpha$-methylene- $\gamma$-butyrolactones," Tetrahedron Letters, vol. 48, no. 18, pp. 3205-3207, 2007.

[8] M. Szlosek-Pinaud, P. Diaz, J. Martinez, and F. Lamaty, "Palladium-catalyzed cascade allylation/carbopalladation/cross coupling: a novel three-component reaction for the synthesis of 3,3-disubstituted-2,3- dihydrobenzofurans," Tetrahedron Letters, vol. 44, no. 48, pp. 8657-8659, 2003.

[9] C. Belaud, C. Roussakis, Y. Letourneux, N. El Alami, and J. Villiéras, "Synthesis of potential cytotoxic $\alpha$-methylene $\gamma$ lactams," Synthetic Communications, vol. 15, no. 14, pp. 12331243,1985 .

[10] E. Ohler, K. Reininger, and U. Schmidt, "A simple synthesis of $\alpha$-methylene $\gamma$-lactones," Angewandte Chemie International Edition, vol. 9, no. 6, pp. 457-458, 1970.

[11] P. Knochel and J. F. Normant, "Addition of functionalized allylic bromides to terminal alkynes," Tetrahedron Letters, vol. 25, no. 14, pp. 1475-1478, 1984.

[12] S. C. Welch and J. M. Gruber, "Synthesis of C-13-substituted retinoic acid analogues," Journal of Organic Chemistry, vol. 47, no. 3, pp. 385-389, 1982.

[13] B. Tarnchampoo, C. Thebtaranonth, and Y. Thebtaranonth, "Addition of functionalized allylic bromides to terminal alkynes," Tetrahedron Letters, vol. 28, no. 52, pp. 6675-6678, 1987.

[14] I. Beltaïef, R. Besbes, H. Amri, and J. Villiéras, “TEAP mediated hydroxylation of allylic bromide: a facile synthesis of 4-methoxycarbonyl-2(5H)-furanone," Tetrahedron Letters, vol. 38, no. 5, pp. 813-814, 1997.

[15] H. Amri and J. Villiéras, "Hydroxyalkylation de la méthylvinylcétone et de l'acrylonitrile en présence de 1, 4-diazabicyclo[2. 2. 2] octane," Tetrahedron Letters, vol. 27, no. 36, pp. 4307-4308, 1986.

[16] R. Besbes, M. Villiéras, and H. Amri, "Improved synthesis and reaction of dimethyl a-(bromomethyl) fumarate with primary amines," Indian Journal of Chemistry B, vol. 36, no. 1, pp. 5-8, 1997.

[17] H. Kraïem, M. I. Abdullah, and H. Amri, "First synthesis of 2[alkylamino(diethoxyphosphoryl)methyl]acrylic ethyl esters," Tetrahedron Letters, vol. 44, no. 3, pp. 553-555, 2003. 
[18] A. Arfaoui and H. Amri, "An effective new access to ethyl 2[(alkylamino)(cyano)methyl] acrylates: first synthesis of ethyl 3-cyano-2-(hydroxymethyl) acrylate," Tetrahedron, vol. 65, no. 25, pp. 4904-4907, 2009.

[19] A. Fray, J. Ben Kraïem, A. Souizi, and H. Amri, "A practical synthesis of diethyl 1-[(alkylamino)(cyano)methyl]vinylphosphonates," Arkivoc, vol. 2012, no. 8, pp. 119-127, 2012.

[20] A. Fray, J. Ben Kraïem, and H. Amri, "Selected reactions of diethyl (E)-1-(bromomethyl)-2-cyanovinylphosphonate with secondary and tertiary amines," Heteroatom Chemistry, vol. 24, no. 6, pp. 460-465, 2013.

[21] M. M. Diaz-Requejo, D. DiSalvo, and M. Brookhart, "Synthesis of 1,2-diheteroatom-substituted alkenes via rhodium-catalyzed intramolecular hydrogen transfer," Journal of the American Chemical Society, vol. 125, no. 8, pp. 2038-2039, 2003.

[22] D. L. Boger, W. L. Corbett, T. T. Curran, and A. M. Kasper, "Inverse electron demand Diels-Alder reactions of N-sulfonyl $\alpha, \beta$-unsaturated imines: a general approach to implementation of the $4 \pi$ participation of 1-aza-1,3-butadienes in Diels-Alder reactions," Journal of the American Chemical Society, vol. 113, no. 5, pp. 1713-1729, 1991.

[23] R. I. Longley Jr. and W. S. Emerson, "The 1,4-addition of vinyl ethers to $\alpha, \beta$-unsaturated carbonyl compounds," Journal of the American Chemical Society, vol. 72, no. 7, pp. 3079-3081, 1950.

[24] H. A. A. El-Nabi, "Novel heterocycles: a convenient synthesis of pyrrolo[2,3-d]pyrazole; cycloaddition reaction of $\mathrm{N}$ aryl(methyl)pyrrol-2,3-diones to diazomethane and olefins," Tetrahedron, vol. 53, no. 5, pp. 1813-1822, 1997.

[25] R. Bruckner and R. Huisgen, "Kinetics of [2+2] cycloadditions of 2,2-bis(trifluoromethyl)ethylene-1,1- dicarbonitrile with enol ethers, 1,1-dimethylbutadiene, and allytrimethylsilane," Tetrahedron Letters, vol. 31, no. 18, pp. 2557-2560, 1990.

[26] S. Futamura, H. Ohta, and Y. Kamiya, "The photocycloaddition of 6-substituted phenanthridines to electron-rich olefins," Bulletin of the Chemical Society of Japan, vol. 55, no. 7, pp. 21902194, 1982.

[27] O. E. O. Hormi and L. Hirvela, "New synthetic approaches to 6thiophenoxysalicylates, 6-phenoxysalicylates and 1-hydroxy-9xanthones," Tetrahedron Letters, vol. 34, no. 40, pp. 6463-6466, 1993.

[28] M. Hojo, R. Masuda, and E. Okada, "A convenient synthetic route to functionalized 5-trifluoroacetyl-6-trifluoromethyl-3, 4-dihydro-2H-pyrans: hetero-diels-alder reaction of $\beta, \beta$ Bis(trifluoroacetyl)vinyl ethers with electron-rich alkenes," Synthesis, vol. 1990, no. 4, pp. 347-350, 1990.

[29] J. Furukawa, N. Kawabata, and J. Nishimura, "Synthesis of cyclopropanes by the reaction of olefins with dialkylzinc and methylene iodide," Tetrahedron, vol. 24, no. 1, pp. 53-58, 1968.

[30] A. de Meijere, T.-J. Schulz, R. R. Kostikov, F. Graupner, T. Murr, and T. Bielfeldt, "Dirhodium(II) tetraacetate catalyzed (chlorovinyl)cyclopropanation of enol ethers and dienol ethers-a route to donor-substituted vinylcyclopropanes, ethynylcyclopropanes and cycloheptadienes," Synthesis, no. 7, pp. 547560, 1991.

[31] E. Wenkert, M. E. Alonso, B. L. Buckwalter, and E. L. Sanchez, "Short syntheses of furan and catechol derivatives. A synthesis of hydrourushiol," Journal of the American Chemical Society, vol. 105, no. 7, pp. 2021-2029, 1983.

[32] A. N. Ajjou and H. Alper, "A new, efficient, and in some cases highly regioselective water-soluble polymer rhodium catalyst for olefin hydroformylation," Journal of the American Chemical Society, vol. 120, no. 7, pp. 1466-1468, 1998.
[33] S. Matysiak, H.-P. Fitznar, R. Schnell, and W. Pfleiderer, "Acetals as new 2'-O-protecting functions for the synthesis of oligoribonucleotides: synthesis of uridine building blocks and evaluation of their relative acid stability," Helvetica Chimica Acta, vol. 81, no. 8, pp. 1545-1566, 1998.

[34] K. Kojima, M. Sawamoto, and T. Higashimura, "Living cationic polymerization of isobutyl vinyl ether by hydrogen iodide/lewis acid initiating systems: effects of lewis acid activators and polymerization kinetics," Macromolecules, vol. 22, no. 5, pp. 1552-1557, 1989.

[35] M. W. Briscoe, R. D. Chambers, S. J. Mullins, T. Nakamura, and J. F. S. Vaughan, "Reactions involving fluoride ion. Part 39. Reactions of perfluorinated dienes with oxygen and sulphur nucleophiles," Journal of the Chemical Society, Perkin Transactions 1, no. 21, pp. 3119-3124, 1994.

[36] A. E. Feiring and E. R. Wonchoba, "Addition of phenols to perfluorovinyl ethers. Protonation and halogenation of carbanionic intermediates," Journal of Organic Chemistry, vol. 57, no. 26, pp. 7014-7017, 1992.

[37] D. Gopal and K. Rajagopalan, "Studies on the sequential claisen rearrangement of methyl-3-arlyloxy- 2-(aryloymethyl)prop-2enolates," Tetrahedron Letters, vol. 28, no. 44, pp. 5327-5330, 1987.

[38] K. Mizuno, Y. Kimura, and Y. Otsuji, "A convenient synthesis of aryl vinyl ethers by use of tetra-n-butylammonium hydrogen sulfate," Synthesis, vol. 1979, no. 9, pp. 688-689, 1979.

[39] M. Blouin and R. Frenette, "A new method for the preparation of aryl vinyl ethers," Journal of Organic Chemistry, vol. 66, no. 26, pp. 9043-9045, 2001.

[40] M. Solinas, S. Gladiali, and M. Marchetti, "Hydroformylation of aryloxy ethylenes by $\mathrm{Rh} / \mathrm{BINAPHOS}$ complex: catalyst deactivation path and application to the asymmetric synthesis of 2aryloxypropanoic acids," Journal of Molecular Catalysis A, vol. 226, no. 1, pp. 141-147, 2005.

[41] C.-M. Andersson and A. Hallberg, "Synthesis of $\beta$-arylvinyl ethers by the palladium-catalyzed reaction of aroyl chlorides with vinyl ethers," Journal of Organic Chemistry, vol. 53, no. 2, pp. 235-239, 1988.

[42] A. K. Filippova, Y. L. Frolov, G. S. Lyashenko et al., "Synthesis and spectroscopic investigation of aryloxy- and arylthioalkenes, alkynes, and allenes," Bulletin of the Academy of Sciences of the USSR Division of Chemical Science, vol. 35, no. 8, pp. 1677-1680, 1986.

[43] J. V. Crivello and S. Kong, "Efficient isomerization of allyl ethers and related compounds using pentacarbonyliron," Journal of Organic Chemistry, vol. 63, no. 19, pp. 6745-6748, 1998.

[44] Y. Okimoto, S. Sakaguchi, and Y. Ishii, "Development of a highly efficient catalytic method for synthesis of vinyl ethers," Journal of the American Chemical Society, vol. 124, no. 8, pp. 1590-1591, 2002.

[45] M. Taillefer, A. Ouali, B. Renard, and J.-F. Spindler, "Mild copper-catalyzed vinylation reactions of azoles and phenols with vinyl bromides," Chemistry, vol. 12, no. 20, pp. 5301-5313, 2006.

[46] A. Ramazani, A. Safari, and N. Noshiranzadeh, "Reaction between dimethyl acetylenedicarboxylate and phenols in the presence of dipotassium hydrogen phosphate in solvent-free conditions: an efficient environment-friendly method for the synthesis of aryl vinyl ethers," Scientia Iranica, vol. 16, no. 1, pp. 7-11, 2009.

[47] R. Baharfar and S. M. Vahdat, "An efficient and green approach for the synthesis of vinyl aryl ethers in the presence of guanidine 
hydrochloride," Turkish Journal of Chemistry, vol. 34, no. 6, pp. 869-874, 2010.

[48] M. R. Kuram, M. Bhanuchandra, and A. K. Sahoo, "Goldcatalyzed intermolecular hydrophenoxylation of unactivated Internal alkynes," Journal of Organic Chemistry, vol. 75, no. 7, pp. 2247-2258, 2010.

[49] M. S. Kabir, M. Lorenz, O. A. Namjoshi, and J. M. Cook, "First application of an efficient and versatile ligand for coppercatalyzed cross-coupling reactions of vinyl halides with $\mathrm{N}$ heterocycles and phenols," Organic Letters, vol. 12, no. 3, pp. 464-467, 2010.

[50] A. M. Klos, G. R. Heintzelman, and S. M. Weinreb, "A $\beta$ hydroxyethyl carbanion equivalent," Journal of Organic Chemistry, vol. 62, no. 11, pp. 3758-3761, 1997.

[51] D. F. Taber, J. H. Green, and J. M. Geremia, "Carbon-carbon bond formation with allylmagnesium chloride," Organic Chemistry, vol. 62, no. 26, pp. 9342-9344, 1997.

[52] A. Inoue, K. Kitagawa, H. Shinokubo, and K. Oshima, "Selective halogen-magnesium exchange reaction via organomagnesium ate complex," Journal of Organic Chemistry, vol. 66, no. 12, pp. 4333-4339, 2001.

[53] N. M. Heron, J. A. Adams, and A. H. Hoveyda, "Stereoselective heteroatom-assisted allylic alkylation of cyclic ethers with Grignard reagents. A convenient route to enantiomerically pure carbocycles," Journal of the American Chemical Society, vol. 119, no. 26, pp. 6205-6206, 1997.

[54] B. H. Lipshutz and S. Sengupta, "Organocopper reagents: substitution, conjugate addition, carbo/metallo-cupration, and other reactions," Organic Reactions, vol. 41, pp. 135-631, 1992.

[55] I. Beltaïef, R. Besbes, F. B. Amor, H. Amri, M. Villiéras, and J. Villiéras, "(Z)-dimethyl $\alpha$-(bromomethyl)fumarate, an efficient intermediate for the selective synthesis of dimethyl 3-alkyl itaconates and 2-alkyl 3- carbomethoxy- $\gamma$-lactams," Tetrahedron, vol. 55, no. 13, pp. 3949-3958, 1999. 

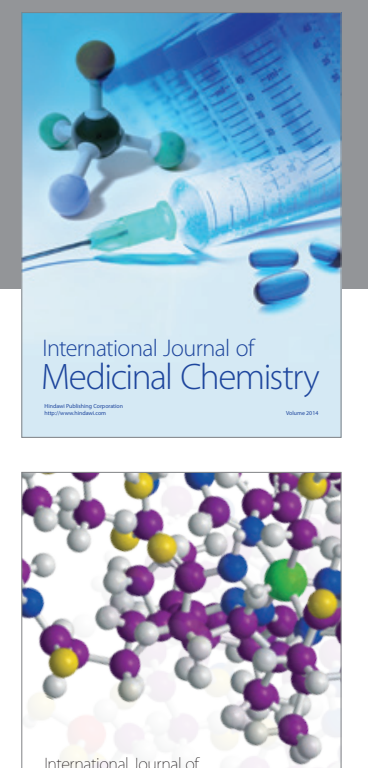

\section{Carbohydrate} Chemistry

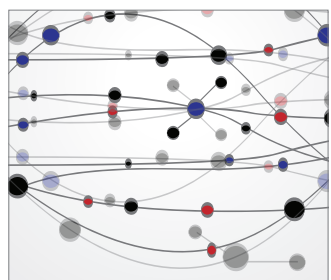

The Scientific World Journal
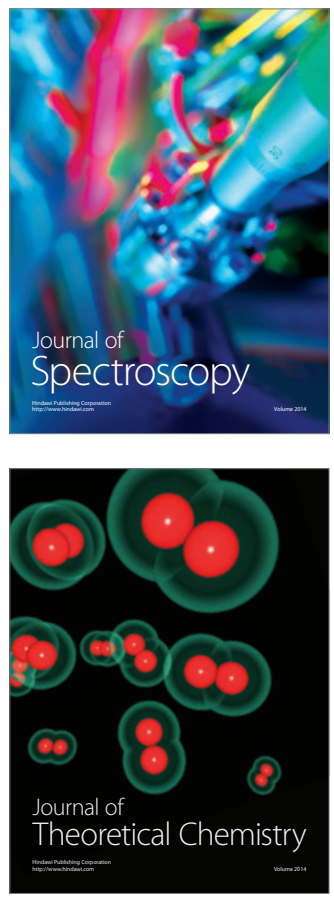
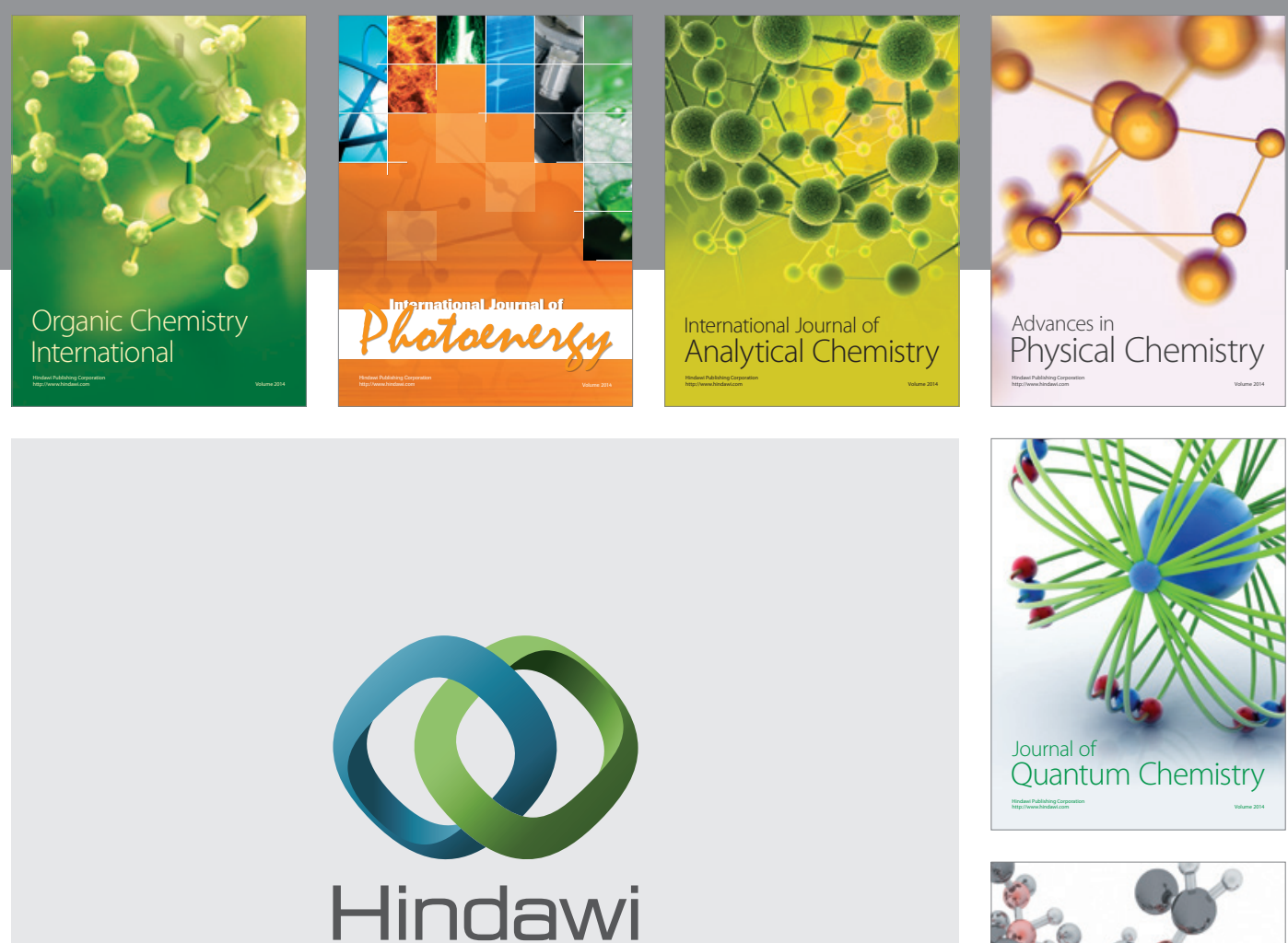

Submit your manuscripts at

http://www.hindawi.com

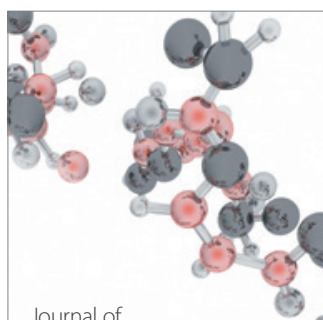

Analytical Methods

in Chemistry

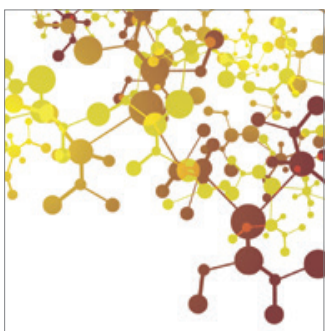

Journal of

Applied Chemistry

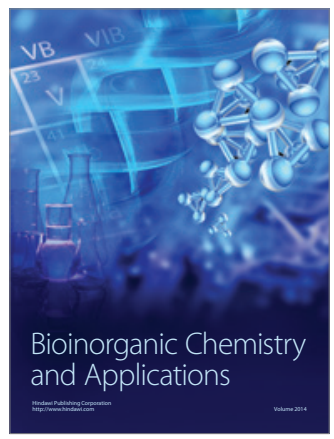

Inorganic Chemistry
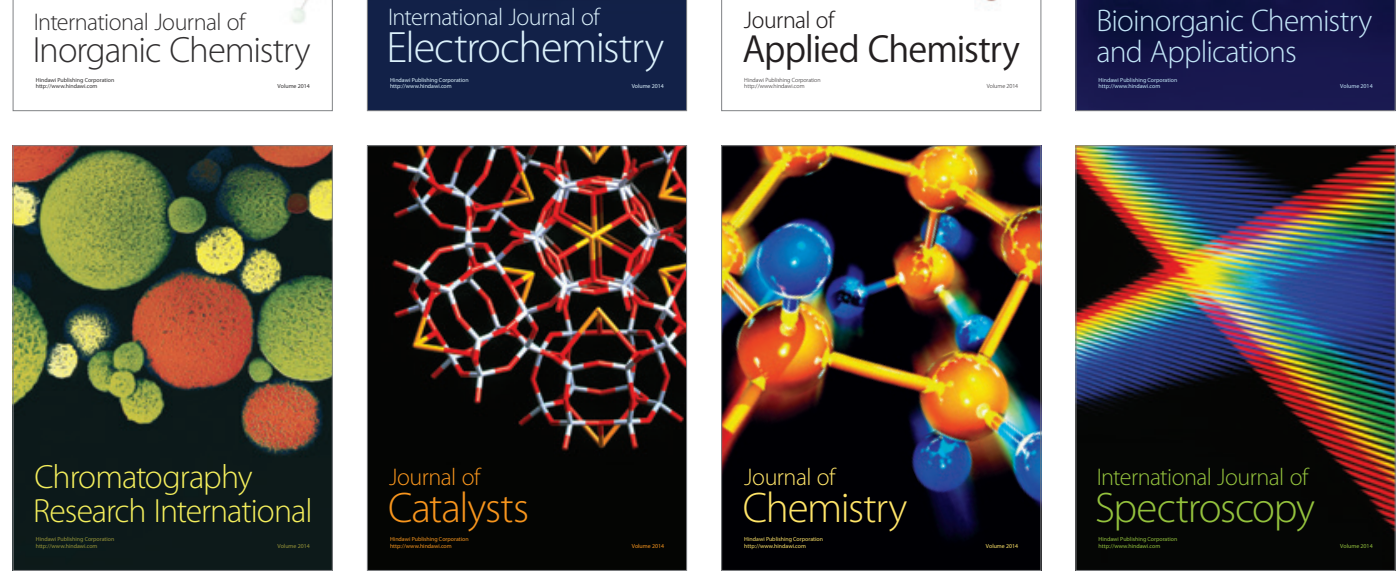